\title{
Luminescence and Crystalline Properties of InGaN-based LED on Si Substrate with AIN/GaN Superlattice Structure
}

\author{
Ezzah Azimah Alias, ${ }^{1}$ Muhamad Ikram Md Taib, ${ }^{1}$ Ahmad Shuhaimi Abu Bakar, ${ }^{2 *}$ \\ Takashi Egawa, ${ }^{3}$ Anthony J. Kent, ${ }^{4}$ Wan Maryam Wan Ahmad Kamil, ${ }^{5}$ and Norzaini \\ Zainal $^{1^{*}}$ \\ ${ }^{1}$ Institute of Nano Optoelectronics Research and Technology (INOR), Universiti Sains \\ Malaysia, 11800 Pulau Pinang, Malaysia \\ ${ }^{2}$ Low Dimensional Materials Research Centre, Department of Physics, Faculty of \\ Science, University of Malaya, Lembah Pantai, 50603 Kuala Lumpur, Malaysia \\ ${ }^{3}$ Research Centre for Nano-device and System, Nagoya Institute of Technology, \\ Gokiso-cho, Showa-ku, Nagoya 466-8555, Japan \\ ${ }^{4}$ School of Physics and Astronomy, University of Nottingham, Nottingham NG7 2RD, \\ United Kingdom \\ ${ }^{5}$ School of Physics, Universiti Sains Malaysia, 11800 Pulau Pinang, Malaysia \\ *Corresponding authors: shuhaimi@um.edu.my, norzaini@usm.my
}

Published online: 25 November 2021

To cite this article: Alias, E. A. et al. (2021). Luminescence and crystalline properties of InGaN-based LED on Si substrate with AlN/GaN superlattice structure. J. Phys. Sci., 32(3), 1-11. https://doi.org/10.21315/jps2021.32.3.1

To link to this article: https://doi.org/10.21315/jps2021.32.3.1

\begin{abstract}
A crack-free indium gallium nitride (InGaN) based light emitting diode (LED) grown on silicon (Si) substrate was successfully demonstrated by introducing aluminium nitride/gallium nitride (AlN/GaN) superlattice structure (SLS) in the growth of the LED. The luminescence and the crystalline properties of the LED were discussed. From photoluminescence (PL) surface mapping measurement, the emission wavelength of the LED (453 nm) was almost uniform across the LED epi-wafer area. Temperaturedependent PL revealed that the dominant emission peak of the LED was $2.77 \mathrm{eV}$ at all temperatures. The emission peak was related to the quantum wells of the LED. Some additional peaks were also observed, in particular at lower temperatures. These peaks were associated to alloy fluctuations in the In $0.11 \mathrm{Ga} 0.89 \mathrm{~N} / \mathrm{In} 0.02 \mathrm{Ga} 0.98 \mathrm{~N}$ multiquantum wells (MQWs) of the LED. Furthermore, the dependence of PL intensity and PL decay time on temperature revealed the evidence related to indium and/or interface fluctuations of the quantum wells. From X-ray diffraction (XRD) $\omega$-scan measurements, fringes of the AlN/GaN SLS were clear, indicating the SLS were grown with good interface abruptness. However, the fringes for the MQWs were less uniform, indicating another evidence of the
\end{abstract}


alloy fluctuations in the MQWs. XRD-reciprocal surface mapping (RSM) measurement showed that all epitaxial layers of the LED were grown coherently, and the LED was fully under strain.

Keywords: photoluminescence, X-ray diffraction, alloy fluctuations

\section{INTRODUCTION}

Silicon $(\mathrm{Si})$ has been considered as a potential substrate for indium gallium nitride (InGaN) based light emitting diodes (LEDs) to reduce production cost. In comparison to sapphire, $\mathrm{Si}$ substrates are cheaper and available in larger diameters (up to $450 \mathrm{~mm}$ ). Furthermore, $\mathrm{Si}$ is a conductive material and therefore, gives a better current injection to the LEDs. Nonetheless, Si also suffers from drawbacks. Large mismatch in terms of lattice constant and thermal expansion coefficient between nitrides and Si materials causes cracks and high threading dislocations in the LEDs, which significantly degrade the overall LEDs performance. ${ }^{1}$ To date, many attempts have been done to reduce the impacts from the mismatch. The most common one is by introducing superlattice structure (SLS) in the LEDs growth. ${ }^{2,3}$ Mainly because such structure can promote inclination of threading dislocations and hence, their annihilations, which in turn, reduces the number of the dislocations from propagating into the multiquantum wells (MQWs) active region of the LED. At this point, the luminescence and crystalline properties of LEDs grown with SLS have not received much attention. Hence, in this work, the luminescence, and crystalline properties of an InGaN-based LED grown with aluminium nitride/gallium nitride (AlN/GaN) SLS will be discussed through a series of photoluminescence (PL) and X-ray diffraction (XRD) measurements.

\section{EXPERIMENTAL}

InGaN-based LED was grown on Si (111) substrate in metal-organic chemical vapour deposition (MOCVD) reactor. The reactor was made by Taiyo Nippo Sanso, based in Tokyo, Japan. Trimethylgallium (TMGa), trimethylaluminum (TMAl), trimethylindium (TMIn) and ammonia $\left(\mathrm{NH}_{3}\right)$ were used as the precursors for $\mathrm{Ga}$, $\mathrm{Al}$, In and $\mathrm{N}$, respectively. Meanwhile, monosilane and bis (cyclopentadienyl) magnesium were used for the Si and $\mathrm{Mg}$ doping. All sources were from Sumitomo Chemical Co., Ltd, Tokyo, Japan. The growth started with a $25 \mathrm{~nm}$ thick AlN layer on the Si substrate. Then, 40 pairs of AlN $(5.0 \mathrm{~nm}) / \mathrm{GaN}(20.0 \mathrm{~nm}) \mathrm{SLS}$ were grown followed by the growth of $\mathrm{n}-\mathrm{GaN}$ layer and of 15 -pairs of $\mathrm{In}_{0.11} \mathrm{Ga}_{0.89} \mathrm{~N}$ $(0.6 \mathrm{~nm}) / \mathrm{In}_{0.02} \mathrm{Ga}_{0.98} \mathrm{~N}(10.0 \mathrm{~nm}) \mathrm{MQWs}$. The growth temperature for the MQWs was at about $800^{\circ} \mathrm{C}$. Subsequently, the p-AlGaN blocking layer and $\mathrm{p}-\mathrm{GaN}$ were grown. 
Next, the luminescence properties of the LED were investigated through PL surface mapping, temperature-dependent PL, and time-resolved PL (TRPL) measurements. The PL surface mapping measurement was performed in Nagoya, Japan, while temperature-dependent PL and TRPL measurements was completed in Nottingham, United Kingdom. From the PL surface mapping measurement, the uniformity of the emission wavelength across the LED epi-wafer area was determined. To understand the thermally activated optical process of carriers in the MQWs, the activation energy was estimated by the temperature-dependent PL measurement. The TRPL measurement was performed to measure the PL decay time at different emission energies. The PL surface mapping measurement was performed at $300 \mathrm{~K}$ using a He-Cd laser with an excitation wavelength of $325 \mathrm{~nm}$. For the temperature-dependent PL and TRPL measurements, a titanium (Ti)-sapphire laser with a wavelength of $266 \mathrm{~nm}$ operating at $5 \mathrm{~mW}$ was used.

The crystalline properties of the LED were investigated using high resolution X'Pert Pro MRD XRD measurement with $\mathrm{Cu}-\mathrm{K} \alpha 1$ radiation source $(\lambda=$ $1.5406 \AA$ ). The XRD system was made by PANalytical, based in Almelo, the Netherlands. XRD $\omega$-scan in (004) reflection was performed to estimate the indium composition and thickness of the quantum wells and quantum barriers of the MQWs as well as the thickness for the SLS. The growth coherency and strain characteristics of the LED was measured via reciprocal surface mapping (RSM) scan.

\section{RESULTS AND DISCUSSION}

The grown InGaN-based LED on $\mathrm{Si}$ with $\mathrm{AlN} / \mathrm{GaN}$ SLS showed a mirrorlike surface and no micro-cracks were observed under optical microscope. Figure 1(a) shows the grown LED structure and Figure 1(b) shows a scanning electron microscopy (SEM) cross-sectional image of the LED.

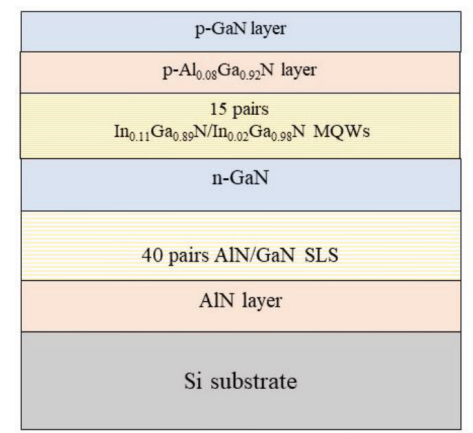

(a)

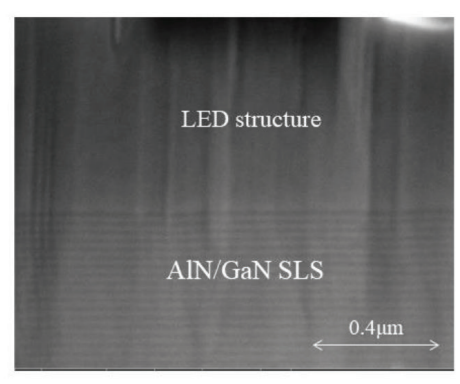

(b)

Figure 1: (a) InGaN-based LED on Si substrate with AIN/GaN SLS and (b) SEM crosssectional image of the LED alongside the SLS. 
Figure 2 shows room-temperature PL surface mapping for the emission wavelength of the InGaN-based LED grown on $\mathrm{Si}$ with AlN/GaN SLS. As shown in Figure 2, the emission wavelength of the LED is almost uniform across the epiwafer area with an average wavelength of $453 \mathrm{~nm}$. In the areas near to or at the edge, the emission wavelength is longer, especially at the orientation flat (OF). During the growth of MQWs, the temperature at the edge was always lower than that at the centre due to limited heat distribution towards the edge. However, at lower temperatures, more indium was incorporated into the InGaN quantum wells, resulting in longer emission wavelength.

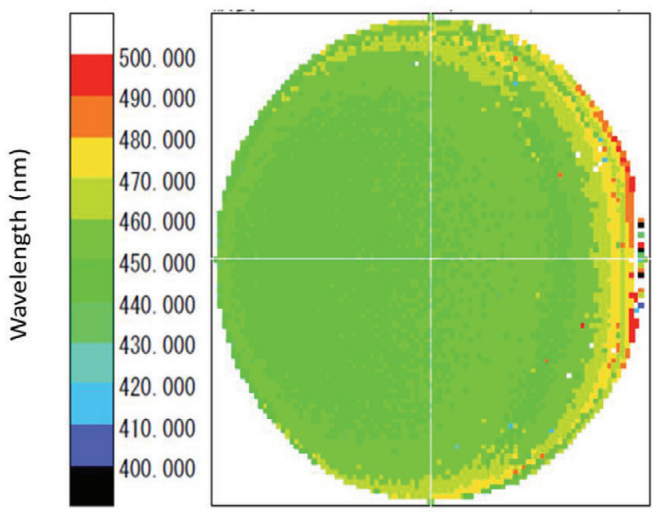

Figure 2: PL surface mapping of emission wavelength for InGaN-based LED with AIN/ GaN SLS.

Subsequently, Figure 3 shows the PL spectra of the LED at measured temperatures from $4 \mathrm{~K}$ to $300 \mathrm{~K}$. Four distinct emission peaks were observed. The peak at $\sim 2.77 \mathrm{eV}$ (defined by peak A) was associated to the emissions from the InGaN quantum wells. Meanwhile, the peaks at $\sim 2.87 \mathrm{eV}$ (defined by peak B) and $\sim 2.99 \mathrm{eV}$ (defined by peak C) were emissions from other InGaN materials due to alloys fluctuation in the MQWs. Such InGaN materials had poor crystalline structure. At higher temperatures, both peaks gradually disappear. This is because at higher temperatures, the non-radiative recombination becomes dominant, especially for poor crystalline alloys. A weak peak at $\sim 3.15 \mathrm{eV}$ (defined by peak D) was due to the barrier layers. 


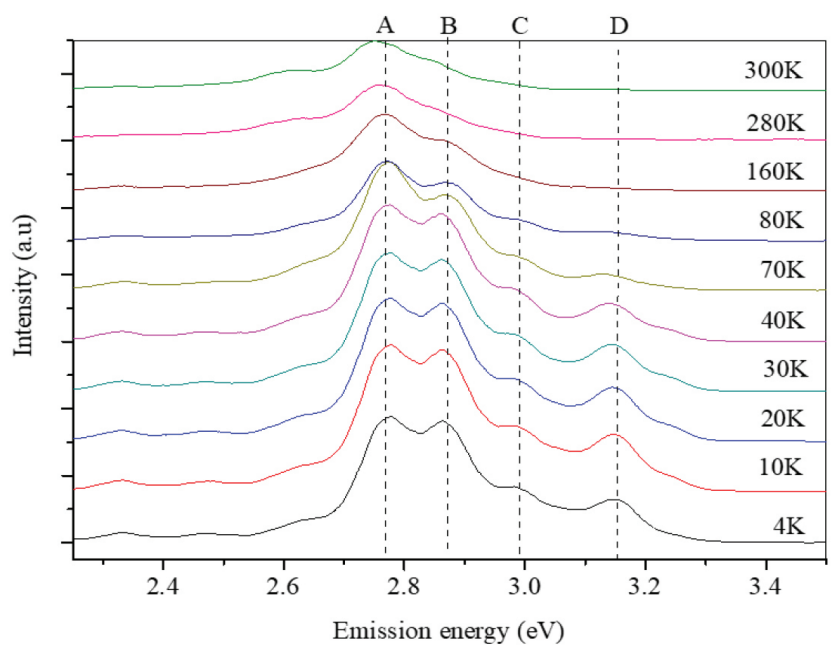

Figure 3: PL intensity as a function of emission energy for InGaN-based LED with AIN/ GaN SLS.

From the results, the internal quantum efficiency (IQE) was estimated by the ratio of the integral intensity at $300 \mathrm{~K}$ to the one at $4 \mathrm{~K}\left(\mathrm{I}_{300 \mathrm{k}} / \mathrm{I}_{4 \mathrm{k}}\right)$, assuming that the efficiency was $100 \%$ at $4 \mathrm{~K}$. The IQE of the LED was $~ 39 \%$ and this value was higher than some InGaN-based LEDs grown on sapphire. ${ }^{4}$ This indicates that the introduction of the AIN/GaN SLS in the LED structure is beneficial to achieve good IQE.

The emission energy of the InGaN quantum wells (peak A) as a function of temperature is shown in Figure 4. The figure includes Varshni fitting that assumes the energy bandgap is shrinking with temperature. ' $S$-shape' characteristic (redshift-blueshift-redshift) can be observed from the figure, which has been widely reported in many works. ${ }^{6}$ In detail, when the temperature increased from $4 \mathrm{~K}$ to $40 \mathrm{~K}$, the emission energy shifted to lower energy sides (red-shift). In this range, the radiative recombination was still dominant. Correspondingly, the carriers had enough time to move and recombine at lower energy tail states. ${ }^{6}$ This increased the portion of the lower emission energies of peak A and hence, the peak was red shifted. The existence of the tail states was due to the inhomogeneous potential of the quantum wells, which originated from indium and/or interface fluctuations. In the temperature range of above $40 \mathrm{~K}$ to $100 \mathrm{~K}$, the carriers' lifetime decreased and therefore, they recombined before reaching the lower energy tail states. As a result, the portion of higher emission energies increased, and blue shift of the peak position was observed. In this range, the result did not follow the Varshni fitting. At temperatures above $100 \mathrm{~K}$, the emission energy showed red-shift behaviour. 
Under this condition, the non-radiative recombination became dominant and the carriers' lifetime was almost constant. Moreover, the trend followed the Varshni fitting.

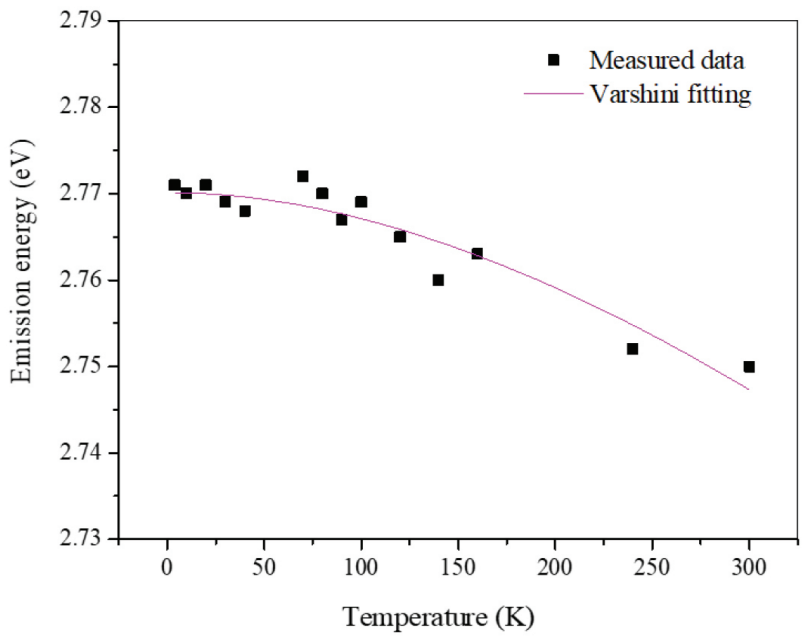

Figure 4: Emission energy of InGaN quantum wells (peak A) for InGaN-based LED with AlN/GaN SLS as a function of temperature.

The integrated PL intensity of the emission energy of InGaN quantum wells (peak A) as a function of temperature is shown in Figure 5(a). From Figure 5(a), the intensity of the PL increased by increasing the temperature from $4 \mathrm{~K}$ to $40 \mathrm{~K}$. Within this range, the number of electrons and holes which recombined radiatively was high due to the longer decay time (lifetime) of the carriers. ${ }^{6}$ Hence, the radiative recombination was significant at lower temperatures and led to increasing the PL intensity. As the temperature was raised up to $140 \mathrm{~K}$, the PL intensity decreased since the decay time was faster. ${ }^{6}$ Thus, non-radiative recombination started to take place. From $140 \mathrm{~K}$ to $240 \mathrm{~K}$, the PL intensity increased with temperature. This could be due to enhancement of carrier mobility by the carrier screening effect of ionised impurity or of defect scattering, which promoted more carriers to be captured by the radiative recombination centres. ${ }^{7}$ Non-radiative recombination became dominant at temperatures above $240 \mathrm{~K}$ and hence, decreased the intensity. 

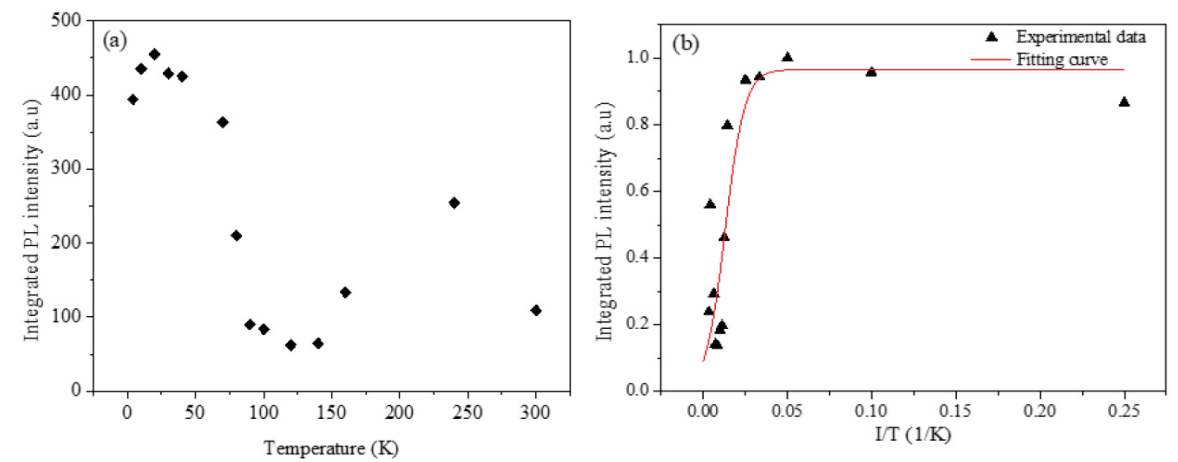

Figure 5: (a) Integrated PL intensity as a function of temperature for InGaN based LED with AlN/GaN SLS and (b) integrated PL intensity as a function of inversed temperature (Arrhenius plot) for InGaN-based LED with AlN/GaN SLS.

The integrated PL intensity as a function of inversed temperature is shown in Figure 5(b). It can be seen that temperature quenching started at $40 \mathrm{~K}$. The activation energy was estimated from the Arrhenius plot through equation (1),

$$
I(T)=\frac{I_{0}}{1+A \exp \left(\frac{-E_{a}}{K_{b} T}\right)}
$$

where, $I(T)$ is the temperature-dependent PL intensity, $I_{0}$ the PL intensity at $0 \mathrm{~K}$ and $K_{b}$ is the Boltzmann constant. $A$ is a constant that corresponds to the ratio of non-radiative recombination and radiative rate recombination in the LED. $E_{a}$ is the activation energy.

From the fitting, the activation energy of the LED was found to be around $15 \mathrm{meV}$. The activation energy is smaller than the offset between the quantum wells and the quantum barriers. This indicates that the thermal quenching was due to thermionic emissions of the carriers, which escaped to the potential minima in the quantum wells. Moreover, at higher temperatures, the activation energy was associated to the non-radiative recombination in the MQWs. However, in our case, the activation energy at high temperatures is relatively low as compared to other reported LEDs. ${ }^{8}$ This could be related to the usefulness of the SLS in reducing the dislocations in the MQWs.

Figure 6 shows the dependence of the PL decay time on the emission energy of the LED, as measured by TRPL at $4 \mathrm{~K}$. It is worth noting that at this temperature, the effect of thermally activated non-radiative recombination is mostly ignored. The integrated PL intensity is also included in the graph. As discussed before, the 
peak emission at $\sim 2.77 \mathrm{eV}$ was coming from the quantum wells, while the peaks at $\sim 2.87 \mathrm{eV}$ and $\sim 2.99 \mathrm{eV}$ were both associated to the alloy fluctuations in the MQWs. The decay time decreased at higher emission energies, but it increased slightly at $\sim 2.87$ and $\sim 2.99 \mathrm{eV}$. Such behaviour has been reported to be indicative of various pathways for radiative recombination to occur, which may exist at higher emissions energies; $\sim 2.87 \mathrm{eV}$ and $\sim 2.99 \mathrm{eV} .{ }^{9}$ The emissions from these pathways could affect the purity of the emission wavelength of the LED.

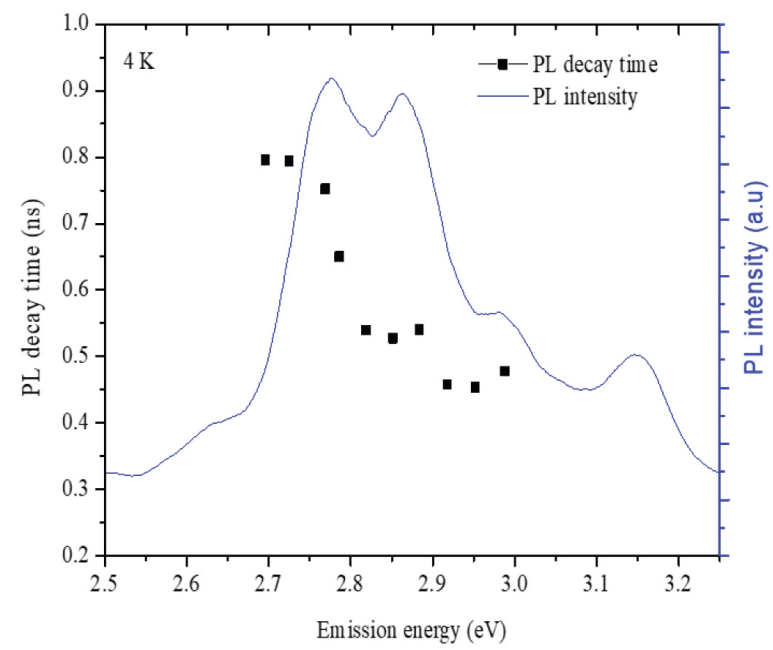

Figure 6: PL spectrum for InGaN-based LED with AIN/GaN SLS at $4 \mathrm{~K}$. Decay time is also included (square dots).

Figure 7 shows XRD $\omega-2 \theta$ scan for the InGaN-based LED with AlN/GaN SLS. The strongest peak is due to the GaN since the LED mostly consists of GaN layers. The fringes at the higher (positive) angle side are corresponding to AlN/ GaN SLS. The appearance of the fringes indicates that the SLS is grown with good abruptness at interfaces. This result is in line with the SEM result [shown in Figure 1(b)], where the periodicity of AlN/GaN SLS is clearly observed with smooth interfaces. The thickness of AIN and GaN of the SLS was $5 \mathrm{~nm}$ and $20 \mathrm{~nm}$, respectively. At the lower (negative) angle side, the fringes are from the MQWs of the LED, which consist of $0.6 \mathrm{~nm}$ thick $\operatorname{In}_{0.11} \mathrm{Ga}_{0.89} \mathrm{~N}$ quantum wells and $20 \mathrm{~nm}$ thick $\operatorname{In}_{0.02} \mathrm{Ga}_{0.98} \mathrm{~N}$ quantum barriers. The fringes, however, are less uniform (the second peak is broader than the first peak), indicating alloy fluctuations in the MQWs. The thicknesses and compositions were determined through a simulation fitting. 


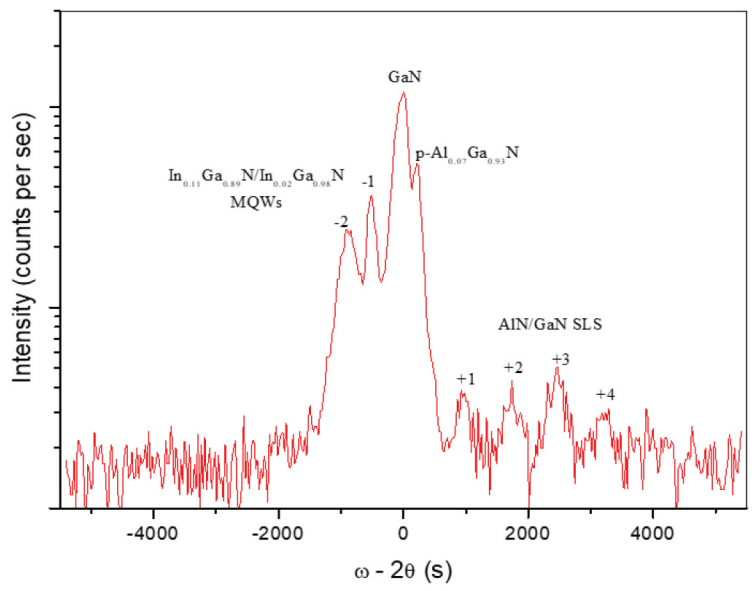

Figure 7: XRD $\omega-2 \theta$ scan for InGaN-based LED with AIN/GaN SLS.

Figure 8 shows XRD-RSM in (002) and (05) reflections. From Figure 8(a), the (002) symmetric scan shows a contour with the highest intensity at the centre, which is contributed from the GaN layer. Meanwhile, the connecting contour at the upper and bottom sides of the GaN layer are due to the AlN/GaN SLS and the $\mathrm{In}_{0.11} \mathrm{Ga}_{0.89} \mathrm{~N} / \mathrm{In}_{0.02} \mathrm{Ga}_{0.98} \mathrm{~N}$ MWQs. It is worth to note that the contour peak of the SLS and the MQWs are well-aligned along the dashed line (the red dashed line). This suggests that the LED is free from relaxation, which means the LED is grown in a good crystalline structure. ${ }^{10}$

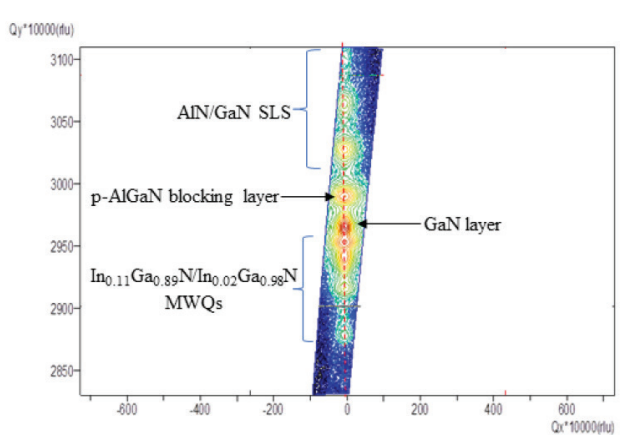

(a)

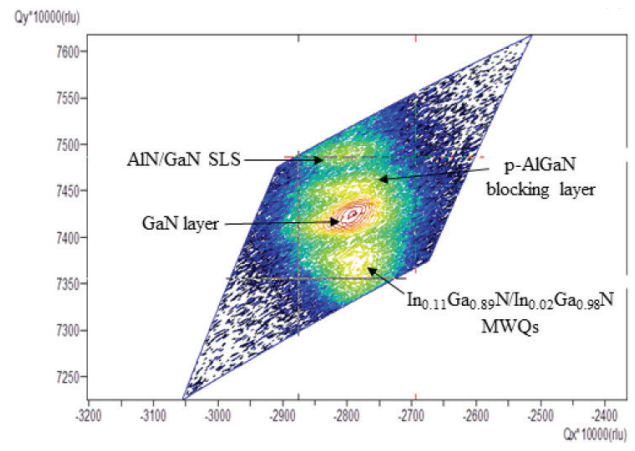

(b)

Figure 8: XRD-RMS for InGaN-based LED with AlN/GaN SLS; (a) (002) symmetric scan and (b) (05) asymmetric scan. 
Figure 8(b) shows the result for the RSM (05) scan for further validation. It can be seen that all the contour peaks are almost coherent to the GaN layer peak, suggesting that the LED is almost fully strained. In general, to obtain a good LED performance, it is essential for all the epitaxial layers in the LED to be grown coherently, meaning fully strain. Otherwise (under relaxation), more defects such as misfit dislocation will be generated. The existence of defects in LEDs would cause serious deterioration to the LED performance. Furthermore, the existence of the strain, particularly in the SLS, force the threading dislocations to incline abruptly. This, in turn, reduces the number of dislocations to reach the MQWs.

\section{CONCLUSION}

From PL measurements, the emission wavelength of the InGaN-based LED on Si with AlN/GaN SLS was almost uniform across the entire area although its MQWs suffered from alloy fluctuations. In addition, the dependence of energy emission and PL intensity on temperature revealed that the quantum wells were grown with indium and/or interfaces fluctuation. At low temperatures, the behaviour of the PL decay time with emission energy was related to the alloy fluctuations in the MQWs, which was further observed in XRD measurements. The measurement also showed that the SLS were grown with good interface abruptness. All epitaxial layers of the LED were grown coherently, and the LED was nearly under strain.

\section{ACKNOWLEDGEMENTS}

This work was supported by the Ministry of Education Malaysia through the Fundamental Research Grant Scheme under account number 203/CINOR/6711718. The authors would like to acknowledge the technical support from DKSH Technology Sdn Bhd and PANalytical Spectris Instrumentation and System for XRD-RSM measurement.

\section{REFERENCES}

1. Yang, Y., Zhang, L. \& Zhao, Y. (2020). Light output enhancement of GaN-based light-emitting diodes based on AlN/GaN distributed Bragg reflectors grown on $\mathrm{Si}$ (111) substrates. Crystals, 10(9), 772. https://doi.org/10.3390/cryst10090772

2. Zhao, X. et al. (2020). Influence of an InGaN superlattice pre-layer on the performance of semi-polar (11-22) green LEDs grown on silicon. Sci Rep., 10, 12650. https://doi.org/10.1038/s41598-020-69609-4 
3. Bin Abu Bakar Ahmad, S., Watanabe, A. \& Egawa, T. (2010). Effect of $\mathrm{Al}_{0.06} \mathrm{Ga}_{0.94} \mathrm{~N} / \mathrm{GaN}$ strained-layer superlattices cladding underlayer to InGaN-based multi-quantum well grown on $\mathrm{Si}(111)$ substrate with AlN/GaN intermediate layer. Jpn. J. Appl. Phys., 49, 021002. https://doi.org/10.1143/JJAP.49.021002

4. Deng, Z. et al. (2013). A novel wavelength-adjusting method in InGaN-based light-emitting diodes. Sci Rep., 3, 3389. https://doi.org/10.1038/srep03389

5. Varshni, Y.P.(1967). Temperature dependence of the energy gap in semiconductors. Physica, 34(1), 149-154. https://doi.org/10.1016/0031-8914(67)90062-6

6. Cho, Y. H. et al. (1998). "S-shaped" temperature-dependent emission shift and carrier dynamics in InGaN/GaN multiple quantum wells. Appl. Phys. Lett., 73(10), 1370. https://doi.org/10.1063/1.122164

7. Lu, T. et al. (2014). Temperature-dependent photoluminescence in light-emitting diodes. Sci Rep., 4, 6131. https://doi.org/10.1038/srep06131

8. Meneghini, M. et al. (2014). Characterization of the deep levels responsible for non-radiative recombination in $\mathrm{InGaN} / \mathrm{GaN}$ light-emitting diodes. Appl. Phys. Lett., 104(11), 113505. https://doi.org/10.1063/1.4868719

9. Lin, T. et al. (2017). Recombination pathways in green $\mathrm{InGaN} / \mathrm{GaN}$ multiple quantum wells. Nanoscale Res. Lett., 12, 137. https://doi.org/10.1186/s11671-0171922-2

10. Meng, Y. et al. (2019). Growth and characterization of amber light-emitting diodes with dual-wavelength InGaN/GaN multiple-quantum-well structures. Mater. Res. Express, 6(8), 0850c8. https://doi.org/10.1088/2053-1591/ab256d 